\title{
Leaf Disease Detection using Deep Learning Algorithm
}

\author{
Kishori Patil, Santosh Chobe
}

\begin{abstract}
India is a nation of agriculture and over 70 per cent of our population relies on farming. A portion of our national revenue comes from agriculture. Agriculturalists are facing loss due to various crop diseases and it becomes tedious for cultivators to monitor the crop regularly when the cultivated area is huge. So the plant disease detection is important in agriculture field. Timely and accurate disease detection is important for the loss caused due to crop diseases which affects adversely on crop quality and yield. Early diagnosis and intervention can reduce the loss of plant due to disease and reduce the unnecessary drug usage. Earlier, automatic detection of plant disease was performed by image processing. For disease detection and classification, image processing tools and the machine learning mechanism are proposed. Crop disease will be detected through various stages of image processing such as image acquisition, pre-processing of image, image feature extraction, feature classification, disease prediction and fertilizer recommendation.detection of disease is important because it will may help farmers to provide proper solution to prevent these disease.
\end{abstract}

Keywords - Classification, Feature Extraction, Image Global Features, Image Processing, Machine Learning.

\section{INTRODUCTION}

Economic growth of farmer depends upon the quality of the yeild that they grow, and which is directly dependent on the growth of the plant and yield they will obtain. Plants are attacked by the different types of disease that target different parts of plant body such as leaf, stem, seed, and fruit and so on. To solve this problem machine learning seems to be a better option.Various machine learning techniques are recently proposed for identification as well as classification of plant disease from plant images. Many crops, most importantly cash crops play a dominant role in the Industrial and Agriculture Economy of the country. India provides direct livelihood to 6 million farmers.

Various image processing concepts such as image filtering, segmentation, image feature extraction have emerged to detect the leaf diseases. There are different image segmentation methods available such as Canny and Sobel segmentation, k-means clustering and Otsu thresholding. For Detection of Cotton Diseases, different techniques such as

Revised Manuscript Received on February 05, 2020.

* Correspondence Author

Kishori Patil, Department of Computer Engineering, Dr. D. Y. Patil Institute of Technology, Savitribai Phule Pune University, Pune, India. Email: kishoripatilshelar@gmail.com

Santosh Chobe, Department of Computer Engineering, Dr. D. Y. Patil Institute of Technology, Savitribai Phule Pune University, Pune, India. Email: sanchobe@gmail.com

(C) The Authors. Published by Blue Eyes Intelligence Engineering and Sciences Publication (BEIESP). This is an open access article under the CC BY-NC-ND license (http://creativecommons.org/licenses/by-nc-nd/4.0/)
Support Vector Machine, Neural Network ,and Homogeneous Pixel Counting can be used for classification. Features play an important role in the process of classification. Previous proposed works for detecting disease have limitations such as low resulting accuracy and less number of images used to detect disease. The plant leaves are the primary cause of the disease. Aroun d 80 to 90 percent of the plant's disease is on plantleaf .Ther efore, the research of interest is the tree's leaf instead of the whole plant. The leaves are mainly suffered from diseases like insecticide (tudtude, mawa), fungus, foliar leaf, and Alternaria leaf spot.

Different types of algorithms are incorporated into the ap plication.Image processing is an important tool for the segm entation of photo into objects and background image. the id entification of the features is one key step in the analysis of $t$ he image .Image recognition has attracted many researchers in the area of pattern recognition, similar flow of concept are applied to the field of pattern recognition of plant leaf, that is used in diagnosing the leaves diseases. There are many methods have been projected in the last two decades which are not fully solved. However this is challenging problems. The critical issue is how to extract the discriminative and stable feature for classification.

\section{REVIEW OF LITERATURE}

Wan Mohd Fadzil et al. [1], mentioned a method that used for detecting disease which occours on leaves of orchid plant. Images of orchid plant leaflet are obtained utilizing digital camera. For categorizing images into two disease class ,aggregate of several strategies like morphological processing ,filtering technique , and border segmentation method are used by the algorithm.. two classes used in this are solar scorch and black leaf spot. However, the segmentation technique proposed and used in this can only distinguish two different types of orchid leaf disease. For classification of other types of leaf disease present on orchid, new or other segmentation technique have to develop. This is because there need many combination of the processing techniques to find robust for border segmentation techniques. Aditya Parikh et al [2] primary focuses on detection of disease and also on estimating disease stage for a given image of cotton plant leaf. The proposed work uses two cascaded classifiers, first classifier segments leaf from the background for which local statistical features are used Then another classifier is trained using luminance and hue $f$ rom HSV colorspace so that classifier can detect disease and identify its level. The algorithm that has been developed is universal, as it can be applied to any disease. However, cascaded classifiers depends on various conditions i.e. border of the leaves are viewable, Leaves are big size for analysis and the probing requires controlled environment.

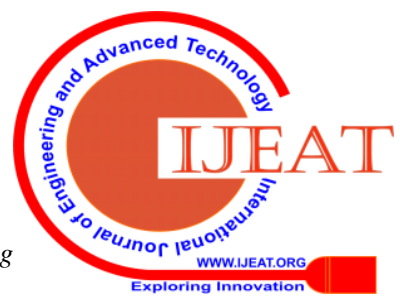


Bhumika S.Prajapati et al [3] presents a survey on cotton leaf disease detection and classification. It is difficult for human eyes to identify exactly which type of leaf disease exists on the plant leaf. Therefore, the usage of machine learning technique and image processing techni que can be helpful to accurately identify the cotton leaf dise ases. The images which are used for this task were acquired using digital camera from the cotton field . In order to remove background from the image the background removal technique is applied in pre-processing step. Then, the background removed images are processed further for image segmentation which is done by otsu thresholding technique. However, this work describe only general and different approach which detect and classify leaf diseases of cotton and describe segmentation as well as background removal techniques.

P. R. Rothe et al [4] presents a pattern recognition system which identify also classify cotton leaf diseases which are Bacterial_Blight, Alternaria and Myrothecium. The pictures taken for this purpose are taken from the cotton fields in Buldana and Wardha district and the fields at "Central Institute of Cotton Research Nagpur". For image segmentation active contour model is used and for the training of adaptive neuro-fuzzy inference system,Hu's moments are extracted as features. However, seven invariant features are extracted from 3 types of diseased_leaves images and this is done to train neuro-fuzzy inference neural network. Neural network classification is depends on invariant features.

Melike Sardogan et al [5] presents a CNN algorithm and Learning Vector Quantization algorithm based method for leaf disease detection and categorization of tomato plant. The dataset contains 500 pictures of tomato plant leaves with four symptoms of diseases. They have modeled a CNN so that automatic feature extraction and classification is done. However, for this study one of the main challenges is that the leaves having different diseases are fundamentally the same as one another. Accordingly, this likeness can make a few leaves be collapsed into to wrong classes.

Norfarahin Mohd Yusoff et al [6] gives a real-time technique of detection of edge for identifying diseases present on Hevea_leaves (rubber leaves) and also its hardware implementation. There are main three diseases which occours on Hevea leaves. for image comparison these three diseses which are Bird's Eye Leaf Spot ,Corynespora Leaf Spot and Collectotrichum Leaf Disease are used. The disease can be detected by using Sobel edge detection algorithm . The result generated by "FPGA Cyclone IV E ".this result is then shown using a monitor. Sobel edge detection algorithm is produce with MATLAB. Result of both techniques are compared. However, Sobel edge_detection algorithm execution depends on MATLAB and FPGA hardware and display the output on the VGA monitor.

Indumathi.R et al [7] discovers the affected area of the leaf, and the disease that attack the leaf as well.This is done with the use of Image Processing. this system make use of " $\mathrm{K}$ Medoid clustering" and the "Random Forest algorithm" to produce greater precision in the leaf disease detection. pre-processing is performed on image, and then the method of clustering is applied to identify the region of the leaf affected. However, Random Forest algorithm is decision tree based algorithm. Accuracy is low compared to other algorithms. Basically random forest used with text data.
Gayatri Kuricheti et al [8] produce an algorithm for detection and prevention of spreading of diseases to the entire crop and results in excellent harvest creation.. The database of various leaf images was created. These images are processed using k-Means image segmentation and textural analysis of leaf images was completed utilizing GLCM. SVM classifier is utilized to categorize the feature extracted images after ranking their attributes using an information gain algorithm. However, the fundamental drawback of K-mean clustering algorithm isthat the number of clusters are required to fix. For the extracting infected part of the leaf , 3 clusters can be ideal.each cluster will denote background in image, healthy part of leaf and the infected part of leaf separately.

Chaowalit Khitthuk et al [9] present a diagnosis system for plantleaf disease utilizing unsupervised neural network. Color features as well as texture features are used for processing images. The system is primarily made of two processes: extraction of features and classification of disease. The method of extraction of the disease feature analyzes the presence of features using statistic-based gray level co-occurrence matrix and texture feature equations.The method of classifying diseases deploys the "unsupervised simplified fuzzy ARTMAP neural network" to categorize disease types. Four forms of photographs of grape leaf disease are used to check the classification efficiency of the method which is rust, downy_mildew, scab and no disease. However in many classifications systems unsupervised feature isn't practically suitable comparing to traditional backpropagation network and machine learning.

PENG JIANG et al [10] presented the collection of images of apple leaves which are affected by disease under real field conditions.complex and laboratory images are used. The dataset is first created via image annotation and data_augmentation technologies. Based on this, a new model that uses deep-CNNs for apple leaf disease detection is proposed by introducing Rainbow concatenation and the Google Net Inception structure. Finally, under the hold-out testing dataset, using a dataset having 26,377 images of diseased apple leaves, the proposed model is trained. This model can be used detecting common apple leaf diseases such as Grey spot, Brown spot, Alternaria leaf spot, Rust and Mosaic. However in this failures of disease detection are observed.

\section{PROPOSED METHODOLOGY}

The process of diagnosis of leaf diseases involves many tasks, such as image_acquisition, preprocessing of image , extraction of features from image and classifying leaf diseases is depends on image feature that is color features, shape features and texture features. The first stage is the image acquisition . In this phase, image is uploaded from the images of the leaf dataset. Then the preprocessing on image is performed using different techniques.

In the third phase, extraction of features is done from the picture for the part of the leaf which is infected.This is done on the basis of particular properties be tween pixels in the image or their texture Then to classify the features which are represent the given image statistical analysis tasks are performed. machine learning is used to compare image features. Finally, classification result

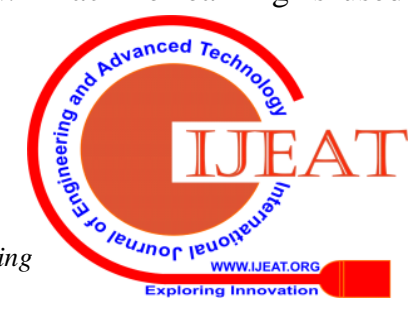


shows the identified leaf disease.

Advantages of proposed system

- It consist of two algorithms for classification and feature extraction which effectively able to extract disease from image and gives the actual final result.

- This proposed system effectively able to extract all the spatial characteristics of an image.

- the detection accuracy using deep learning can be improved.

\section{A. System Architecture}

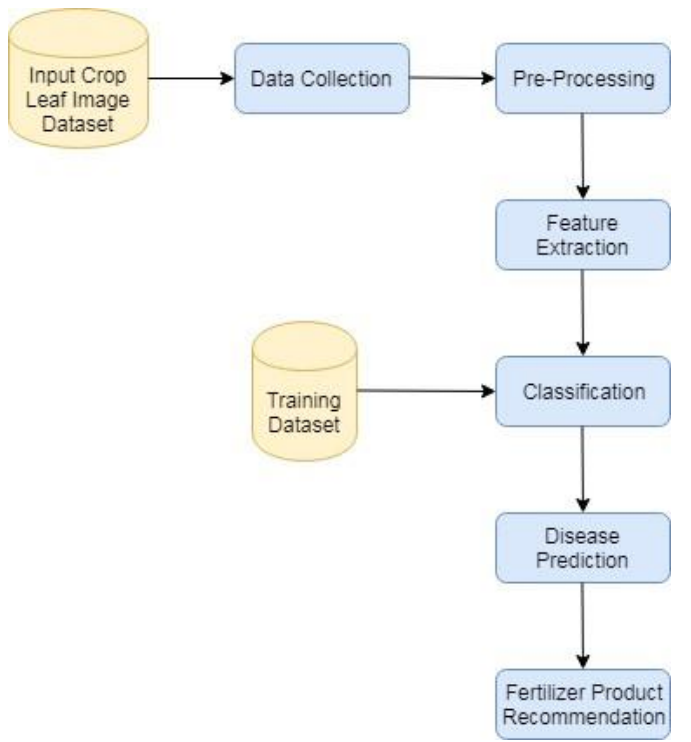

Fig. 1. Proposed System Architecture

\section{B. Mathematical Model}

The mathematical model for Leaf Disease system is as $\mathrm{S}=\{\mathrm{I}, \mathrm{F}, \mathrm{O}\}$

Where,

$\mathrm{I}=$ Set of image leaf dataset

$\mathrm{F}=$ Set of functions

$\mathrm{O}=$ leaf disease prediction

$\mathrm{F}=\{\mathrm{F} 1, \mathrm{~F} 2, \mathrm{~F} 3\}$

F1=Data Collection,

F2=Data Preprocessing,

F3=Feature Selection,

F4=Classifification

F5=Leaf disease detection .

\section{Algorithm}

\section{Convolution Neural Network(CNN) Algorithm:}

The structure of CNN algorithm includes two layers.First is the extraction layer of features in which each neuron's input is directly connected to its previous layer's local ready fields and local features are extracted. The spatial relationship between it and other features will be shown once those local features are extracted. The other layer is feature_map layer; Every feature map in this layer is a plane, the weight of the neurons in one plane are same. The feature plan"s structure make use of the function called sigmoid. This function known as activation function of the CNN, which makes the feature map have shift in difference. In the CNN each convolution layer is come after a computing layer and it's usage is to find the local average as well as the second extract; this extraction of two feature is unique structure which decreases the resolution.

Step 1: Select the dataset.
Step 2: Perform feature selection using information gain and ranking

Step 3: Apply Classification algorithm CNN

Step 4: Calculate each Feature fx value of input layer

Step 5: Calculate bias class of each feature

Step 6: The feature map is produced and it goes to forward pass input layer

Step 7: Calculate the convolution cores in a feature pattern Step 8: Produce sub sample layer and feature value.

Step 9: Input deviation of the kth neuron in output layer is Back propagated.

Step 10: Finally give the selected feature and classification results.

\section{RESULT AND DISCUSSION}

The section shows overall accuracy of CNN classification technique. So this works gives better leaf disease prediction compare to existing method.

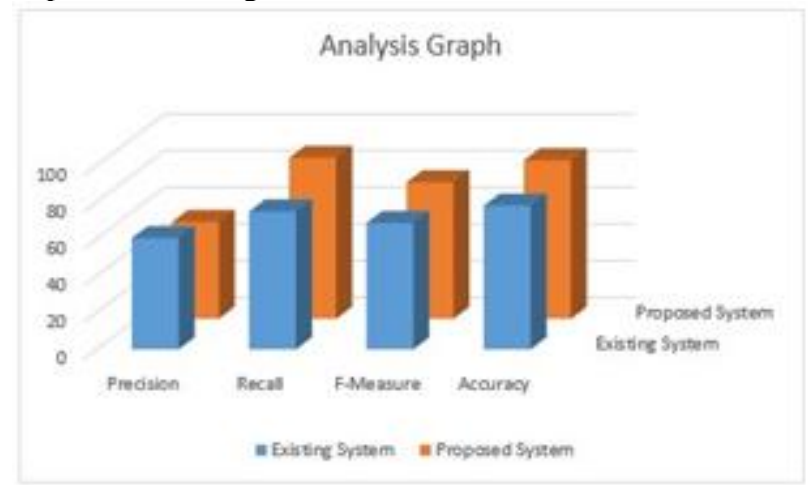

Fig. 2. CNN Classification Accuracy Graph Table No 1.Method Comparison

\begin{tabular}{|c|c|c|}
\hline & Existing System & $\begin{array}{c}\text { Proposed } \\
\text { System(CNN) }\end{array}$ \\
\hline Precision & 60.6 & 52.70 \\
\hline Recall & 75.1 & 87.64 \\
\hline F-Measure & 68.8 & 74.31 \\
\hline Accuracy & 78.29 & 86.26 \\
\hline
\end{tabular}

\section{CONCLUSION}

Here,how the disease analysis is done for the leaf diseases detection is addressed, the analysis of the different diseases that are present on the leaves can be effectively detected in the early stage before it will damage the whole plant. Here the technique presented can able to detect the disease more accurately, we can say that, we can archive good productivity by preventing the different diseases which are present on the leaves of plant using weather dataset and image processing. The usage of classification and feature extraction processes has enhanced the performance of the system which provides better results.

\section{REFERENCES}

1. Chouhan, Siddharth Singh, Ajay Kaul, Uday Pratap Singh, and Sanjeev Jain. "Bacterial foraging optimization based radial basis function neural network (BRBFNN) for identification and classification of plant leaf diseases: An automatic approach towards plant pathology." IEEE Access 6 (2018): 8852-8863.

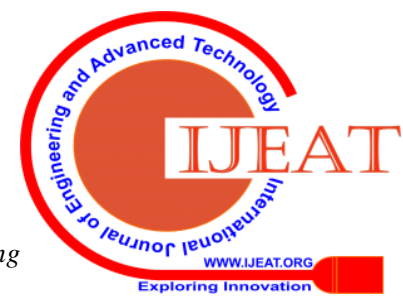


2. Fadzil, WM N. Wan Mohd, MS B. Shah Rizam, R. Jailani, and M. T. Nooritawati. "Orchid leaf disease detection using border segmentation techniques." In 2014 IEEE Conference on Systems, Process and Control (ICSPC 2014), pp. 168-173. IEEE, 2014.

3. Parikh, Aditya, Mehul S. Raval, Chandrasinh Parmar, and Sanjay Chaudhary. "Disease detection and severity estimation in cotton plant from unconstrained images." In 2016 IEEE International Conference on Data Science and Advanced Analytics (DSAA), pp. 594-601. IEEE, 2016.]

4. Prajapati, Bhumika S., Vipul K. Dabhi, and Harshadkumar B. Prajapati. "A survey on detection and classification of cotton leaf diseases." In 2016 International Conference on Electrical, Electronics, and Optimization Techniques (ICEEOT), pp. 2499-2506. IEEE, 2016.

5. Rothe, P. R., and R. V. Kshirsagar. "Cotton leaf disease identification using pattern recognition techniques." In 2015 International Conference on Pervasive Computing (ICPC), pp. 1-6. IEEE, 2015.

6. Sardogan, Melike, Adem Tuncer, and Yunus Ozen. "Plant leaf disease detection and classification based on CNN with LVQ algorithm." In 2018 3rd International Conference on Computer Science and Engineering (UBMK), pp. 382-385. IEEE, $2018 .$.

7. Yusoff, Norfarahin Mohd, Ili Shairah Abdul Halim, and Noor Ezan Abdullah. "Real-time Hevea Leaves Diseases Identification using Sobel Edge Algorithm on FPGA: A Preliminary Study." In 2018 9th IEEE Control and System Graduate Research Colloquium (ICSGRC), pp. 168-171. IEEE, 2018.

8. Indumathi, R., N. Saagari, V. Thejuswini, and R. Swarnareka. "Leaf Disease Detection and Fertilizer Suggestion." In 2019 IEEE International Conference on System, Computation, Automation and Networking (ICSCAN), pp. 1-7. IEEE, 2019.

9. Kuricheti, Gayatri, and P. Supriya. "Computer Vision Based Turmeric Leaf Disease Detection and Classification: A Step to Smart Agriculture." In 2019 3rd International Conference on Trends in Electronics and Informatics (ICOEI), pp. 545-549. IEEE, 2019.

10. Khitthuk, Chaowalit, Arthit Srikaew, Kitti Attakitmongcol, and Prayoth Kumsawat. "Plant Leaf Disease Diagnosis from Color Imagery Using Co-Occurrence Matrix and Artificial Intelligence System." In 2018 International Electrical Engineering Congress (iEECON), pp. 1-4. IEEE, 2018.

11. Jiang, Peng, Yuehan Chen, Bin Liu, Dongjian He, and Chunquan Liang. "Real-Time Detection of Apple Leaf Diseases Using Deep Learning Approach Based on Improved Convolutional Neural Networks." IEEE Access 7 (2019): 59069-59080.

\section{AUTHORS PROFILE}

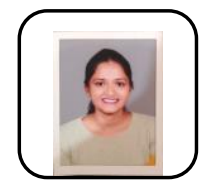

Kishori Patil is persuing ME(Comp) at "DR.D. Y. Patil Institute of Technology", Pimpri, Pune and has completed BE(Comp.Sci.) from Rajiv Gandhi Institute Of Technology, Andheri, Mumbai. Her areas of interests include Data Mining and machine leaning.

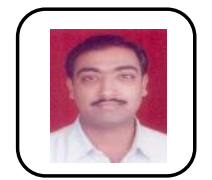

Dr. Santosh V. Chobe has obtained his Bachelors' Degree in Computer Science \& Engineering from D. Y Patil College of Engineering and Technology, Kolhapur, Masters' Degree, ME in Computer Science \& Engineering from Walchand College of Engineering, Sangli and Ph. D. in Computer Engineering from Savitribati Phule Pune University, formerly known as University of Pune.. He is working as Associate Professor in the Computer Engineering Department at Dr. D. Y. Patil Institute of Technology, Pimpri, Pune. He has published more than 30 research papers at the National and International Conferences and Journals. His areas of interests include Data Mining, Web Mining and Compilers. 